\title{
Ofidiotoxicosis por cascabel pigmea (Crotalus ravus): reporte de dos casos en Huixquilucan, Estado de México
}

\author{
Pygmy rattlesnake (Crotalus ravus) ophidiotoxicosis: report \\ of two cases in Huixquilucan, State of Mexico
}

\author{
Yaneli Ayerim González Chávez, * Jorge Guillermo Pérez Tuñón, ${ }^{\ddagger}$ \\ Mayré Ivonne Bautista Albíter, ${ }^{\S}$ Yadira Jodisel Rosales Bacilio ${ }^{\S}$
}

Citar como: González CYA, Pérez TJG, Bautista AMI, Rosales BYJ. Ofidiotoxicosis por cascabel pigmea (Crotalus ravus): reporte de dos casos en Huixquilucan, Estado de México. Acta Med GA. 2022; 20 (1): 83-87. https://dx.doi.org/10.35366/103563

\section{Resumen}

El accidente ofídico es la lesión resultante de la mordedura de una serpiente, posteriormente, si se ha inoculado veneno se desarrollarán las alteraciones locales y/o sistémicas que en su conjunto reciben el nombre de ofidiotoxicosis. En el ámbito mundial, se calcula que cada año se registran 5.4 millones de accidentes ofídicos, lo cual genera de 81,000 a 131,000 muertes por ofidiotoxicosis. En México, durante 2019 se registraron 4,115 mordeduras por serpiente, afectando principalmente a hombres en edad reproductiva; sin embargo, se estima que exista un subregistro considerable en toda la región. Huixquilucan es un municipio del Estado de México que se ubica al poniente de la Ciudad de México. Debido a que la mayor parte de su territorio es ocupado por bosque de coníferas, entre las especies de importancia toxicológica se pueden encontrar vipéridos como Crotalus ravus (cascabel pigmea) y Crotalus triseriatus (cascabel transvolcánica), ya que este entorno representa uno de sus principales hábitats. Presentamos dos casos de pacientes pediátricos que desarrollaron ofidiotoxicosis en el municipio de Huixquilucan en momentos diferentes, los cuales fueron tratados exitosamente con el faboterápico antiviperino.

Palabras clave: Mordedura de serpiente, veneno, faboterapia, Crotalus ravus.

\section{INTRODUCCIÓN}

Se define al accidente ofídico como la lesión resultante de la mordedura de una serpiente, posterior a lo cual, si se ha inoculado veneno se desarrollarán las alteraciones locales

\section{Abstract}

Ophidian accident is the injury resulting from a snake bite, in which if venom has been inoculated, local and/or systemic alterations will develop, these alterations are named ophidiotoxicosis. Worldwide, it is estimated that 5.4 million ophidian accidents are recorded each year, which generates between 81,000 and 131,000 deaths from ophidiotoxicosis. In Mexico, during 2019, 4,115 snake bites were registered, mainly affecting men, however, it is estimated that there is a considerable under-registration throughout the region. Huixquilucan is a municipality in the State of Mexico that is located west of Mexico City. Most of its territory is occupied by coniferous forests, so among the species of toxicological importance can be found viperids such as Crotalus ravus and Crotalus triseriatus, since this environment represents one of its main habitats. We report two pediatric cases with snake envenomation by Crotalus ravus in Huixquilucan at different times, which were successfully treated with fabotherapy.

Keywords: Snake bites, snake venoms, fabotherapy, Crotalus ravus.

y/o sistémicas que en su conjunto reciben el nombre de ofidiotoxicosis o envenenamiento ofídico. ${ }^{1}$

En el ámbito mundial, se calcula que cada año se registran 5.4 millones de accidentes ofídicos, lo cual genera de 81,000 a 131,000 muertes por ofidiotoxicosis y dos
* Residente de segundo año del Postgrado de Alta Especialidad en Toxicología Clínica. México.

₹ Jefe del Centro Toxicológico Hospital Angeles Lomas. México.

§ Toxicóloga Clínica adscrita al Centro Toxicológico Hospital Angeles Lomas. México.
Correspondencia:

Dra. Yaneli Ayerim González Chávez

Correo electrónico:

toxicologia_angeles@yahoo.com.mx

www.medigraphic.com/actamedica 
a tres veces más casos de amputaciones y discapacidad permanente por esta misma causa. Las regiones más afectadas son África, Asia y Latinoamérica. ${ }^{2,3}$ En México, durante 2019 se registraron 4,115 mordeduras por serpiente, afectando principalmente a hombres en edad reproductiva; sin embargo, se estima que exista un subregistro considerable en toda la región. ${ }^{4,5}$ Con respecto al Estado de México, se calculó una incidencia de 1.33 casos por cada 100,000 habitantes en 2018 (225 casos reportados), lo cual lo coloca por debajo de la media nacional (3.09 casos por cada 100,000 habitantes), siendo los municipios de Toluca, Tenancingo, Atlacomulco y Amecameca los más afectados. ${ }^{6}$ Con respecto a la Jurisdicción Sanitaria de Xonacatlán, donde se incluye la totalidad de reportes de accidente ofídico de los municipios de Huixquilucan y Xonacatlán, en el mismo año se reportaron únicamente ocho casos entre las dos entidades.

Huixquilucan es un municipio del Estado de México que se ubica al poniente de la Ciudad de México y forma parte de su área metropolitana. Debido a que la mayor parte de su territorio es ocupado por bosque de coníferas, entre las especies de importancia toxicológica se pueden encontrar vipéridos como Crotalus ravus (cascabel pigmea) y Crotalus triseriatus (cascabel transvolcánica), ya que este entorno representa uno de sus principales hábitats. Adicionalmente, $C$. ravus se distribuye también en los estados de Veracruz, Puebla, Morelos, Oaxaca, Ciudad de México y Tamaulipas. ${ }^{8,9}$

Presentamos el caso de dos pacientes que sufrieron envenenamiento por C. ravus en el municipio de Huixquilucan en momentos diferentes. En ambos casos se logró colectar a los especímenes agresores, los cuales fueron identificados gracias a la colaboración del laboratorio de reptiles del Instituto de Biología de la Universidad Nacional Autónoma de México. Cabe señalar que hasta la fecha no existen reportes en la literatura de ofidiotoxicosis en donde se haya confirmado la participación de C. ravus.

\section{CASO 1}

Paciente masculino de 13 años, con antecedente de retraso leve en el neurodesarrollo, fue llevado a Urgencias una hora después de haber sufrido la mordedura de una serpiente en la mano izquierda en dos ocasiones. A la exploración física, los signos vitales iniciales fueron: frecuencia cardiaca (FC) $96 x^{\prime}$, frecuencia respiratoria (FR) 22 x', tensión arterial (TA) 102/64 mmHg, peso $49.5 \mathrm{~kg}$. Presentó lesiones puntiformes compatibles con huellas de colmillos en el dorso de la mano izquierda (Figura 1), acompañadas de exudado seroso y edema circundante de $17 \mathrm{~cm}$. Se estableció el diagnóstico de ofidiotoxicosis grado 2 y se inició el esquema con faboterápico específico a la dosis recomendada por el fabricante ${ }^{10}$ (Tabla 1), además de que se elevó e inmovilizó la extremidad mediante un vendaje de Jones y se administró analgesia opioide. A las seis horas de evolución presentó progresión del edema a $47 \mathrm{~cm}$ y se incrementó en el valor sérico de la creatincinasa (CK), así como una prolongación del tiempo de protrombina (TP), por lo que se reclasificó el envenenamiento a un grado 3 y se ajustó la dosis de faboterápico. Recibió un total de 50 frascos de faboterápico en una dosis inicial de 10 frascos y cinco dosis subsecuentes de ocho frascos cada una, suspendiéndose al corroborar la corrección de la $\mathrm{CK}$, los tiempos de coagulación y el edema. Fue egresado a su domicilio con apoyo de terapia de rehabilitación por cuatro semanas para mejorar la movilidad de la extremidad afectada.

\section{CASO 2}

Paciente masculino de 15 años, llevado a urgencias 20 minutos después de haber sido mordido por una serpiente en la falange distal del tercer dedo de la mano derecha. A la exploración física, los signos vitales fueron: FC $100 x^{\prime}$, FR $21 x^{\prime}$, TA 100/60 mmHg, peso $54 \mathrm{~kg}$, con aumento de volumen periférico a la lesión de $10 \mathrm{~cm}$, acompañado de equimosis y sangrado escaso (Figura 2). Se integra el diagnóstico de ofidiotoxicosis grado 2 , iniciándose tratamiento con faboterápico específico, analgesia opioide, elevación e inmovilización de la extremidad afectada con vendaje de Jones. Después de la primera dosis, no se observó progresión del edema y los parámetros de laboratorio sólo registraron cambios mínimos (Tabla 2), por lo que se continuó con una dosis de mantenimiento de cinco frascos, de tal

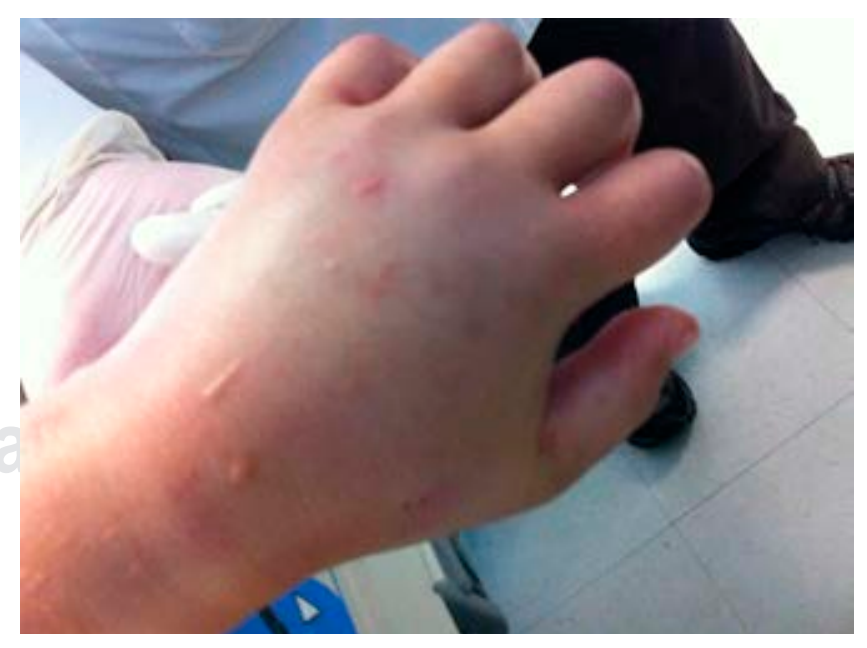

Figura 1: Lesiones puntiformes compatibles con huellas de colmillos en el dorso de la mano izquierda y presencia de edema. 
Tabla 1: Datos clínicos de ofidiotoxicosis, clasificación por severidad y dosis de faboterápico recomendada.

\begin{tabular}{|c|c|c|c|c|c|}
\hline \multirow[b]{2}{*}{ Grado } & \multirow[b]{2}{*}{ Presentación clínica } & \multicolumn{2}{|c|}{ Dosis en niños } & \multicolumn{2}{|c|}{ Dosis en adultos } \\
\hline & & Inicial & Sostén & Inicial & Sostén \\
\hline 1 & $\begin{array}{l}\text { Huellas de colmillos, dolor y edema circundante } \\
\text { de hasta } 10 \mathrm{~cm} \text { de diámetro }\end{array}$ & $6-10$ & 5 & $3-5$ & 5 \\
\hline 2 & $\begin{array}{l}\text { Edema mayor a } 10 \mathrm{~cm} \text {, dolor severo, náusea, vómito, } \\
\text { fasciculaciones, malestar general, ansiedad, debilidad, flictenas } \\
\text { y oliguria. Cambios mínimos en: creatinina, gases arteriales, } \\
\text { creatincinasa, plaquetas, fibrinógeno o tiempos de coagulación }\end{array}$ & 15 & 5 & $6-10$ & 5 \\
\hline 3 & $\begin{array}{l}\text { Necrosis o bulas hemorrágicas, dolor abdominal, disnea, } \\
\text { hipotensión, oligoanuria o anuria, hemorragia distal al sitio de } \\
\text { la mordedura y alteración mayor en uno o más paraclínicos }\end{array}$ & $20-30$ & $10-15$ & $11-15$ & $6-8$ \\
\hline 4 & Choque, disfunción orgánica múltiple y coma & 31 o más & 16 o más & 16 o más & 8 o más \\
\hline
\end{tabular}

modo que fueron requeridos un total de 26 frascos (dosis inicial de seis frascos y cuatro dosis subsecuentes de cinco frascos cada una). Se mantuvo cuatro días en observación y egresó a su domicilio para continuar con terapia de rehabilitación de forma ambulatoria por cuatro semanas, después de esto se reportó una completa recuperación.

\section{DISCUSIÓN}

Los casos fueron elegidos para su reporte debido a las similitudes que presentan en cuanto a la edad de los pacientes, su género, el sitio donde recibieron la mordedura y la especie involucrada con el envenenamiento. Pese a ello, en el primer caso se observó una mayor extensión del edema, así como alteraciones paraclínicas que condujeron a su reclasificación y, por lo tanto, a la administración de una mayor cantidad de faboterápico, lo cual pudo relacionarse con algunos de los factores que se señalan a continuación:

Segunda mordedura: el paciente del caso 1 recibió dos mordeduras en el dorso de la mano, lo que incrementa el tiempo de contacto con los colmillos del espécimen y puede relacionarse con una mayor inoculación de veneno.

Tiempo de inicio del tratamiento: otro aspecto a considerar es que el caso 1 comenzó a recibir el faboterápico luego de una hora de haber ocurrido el accidente, en contraste con el caso 2, que tuvo un tiempo de llegada corto, lo que permitió el inicio del faboterápico antes de los 30 minutos de haber ocurrido la mordedura.

Diseminación del veneno: considerando que el veneno se disemina por vía linfática, el sitio de la mordedura cobra trascendencia debido a que el dorso de la mano (donde ocurrió la lesión del caso 1) se encuentra más vascularizado que las falanges de los dedos (donde ocurrió el caso 2).

Factores indeterminados: en los casos descritos existieron factores que no fue posible determinar, tal es el caso de la cantidad y la concentración del veneno inoculado por ambos especímenes. Tanto la concentración de toxinas en el veneno como la cantidad disponible para su inoculación son características que varían entre individuos de la misma especie y aún en el mismo individuo, dependiendo de su edad, su estado nutricional, el clima de su hábitat, si se

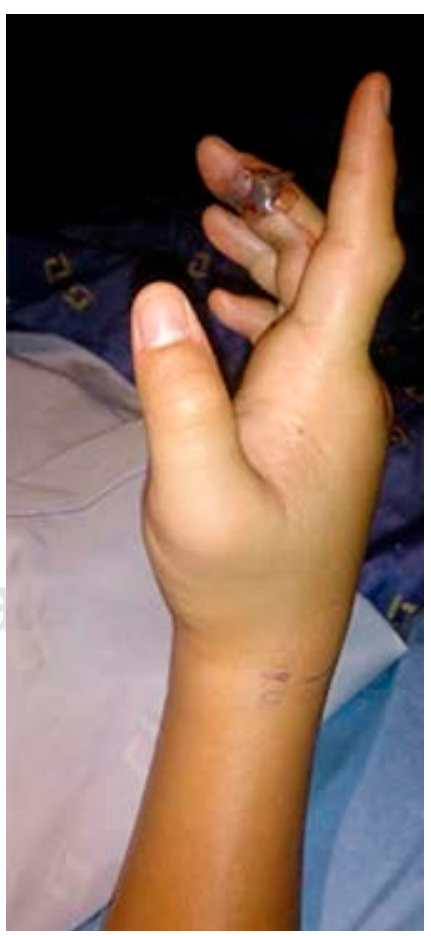

Figura 2:

Edema perilesional, acompañado de equimosis y sangrado escaso en tercer dedo de mano derecha. 
Tabla 2: Evolución clínica.

Tiempo de

evolución

\section{Caso 1}

20 minutos

1 hora

6 horas

14 horas

24 horas

48 horas

72 horas

4 días

1 semana
Edema de $17 \mathrm{~cm}$ con exudado seroso. Hto: 46.6 , PI: 187,000, CK: 149, TP: 13.8, TPT: 27.5, F: 127 Edema de $47 \mathrm{~cm}$

Edema de $40 \mathrm{~cm}$, Hto: 30.5 , PI: 126,000, CK: 5,357, TP: 19.3, TPT: 28.7, F: 136

Edema de $39 \mathrm{~cm}$, Hto: 29.4, PI: 108,000, CK: 6,480, TP: 17.3, TPT: 27.3, F: 242

Edema de $30 \mathrm{~cm}$, Hto: $31.1, \mathrm{Pl}: 125,000, \mathrm{CK}$ :

5,760, TP: 16.7, TPT: 25.7, F: 320

Edema de $21 \mathrm{~cm}$, Hto: 30.5, PI: 123,000, CK:

4,680, TP: 15.4, TPT: 26, F: 362

Edema de $13 \mathrm{~cm}$, consistencia blanda, Hto: 33.3,

PI: 149,000, CK: 3,019, TP: 16.7, TPT: 26.5, F: 333

Sin edema, Hto: 32.6 , PI: 150,000, CK: 110, TP:

14.8, TPT: 27.3, F: 294

\section{Caso 2}

Edema de $10 \mathrm{~cm}$, equimosis y sangrado escaso

$-$

Edema de $7 \mathrm{~cm}$, Hto: 47.5, PI: 329,000, CK: 66, TP: 18.0, TPT: $32.2, \mathrm{~F}: 250$

Edema de $6 \mathrm{~cm}$, Hto: 43.4, PI: 169,000, CK: 85, TP:

13.8, TPT: 27.5, F: 127

Edema de $4 \mathrm{~cm}$, Hto: 45.6, Pl: 174,000, CK: 46, TP:

16.2, TPT: 29.2, F: 399

Edema de $2 \mathrm{~cm}$, Hto: 45.2, PI: 183,000, CK: 49, TP:

15, TPT: 26.8, F: 416

Sin edema, Hto: 46.6, PI: 189,000, CK: 62, TP: 14.4, TPT: 28.7, F: 404

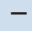

$-$

Hto = hematocrito $(\%) ; \mathrm{PI}=$ plaquetas $(\mathrm{u} / \mu \mathrm{L}) ; \mathrm{CK}=$ creatincinasa $(\mu \mathrm{mol} / \mathrm{L}) ; \mathrm{TP}=$ tiempo de protrombina $(\mathrm{s}) ; \mathrm{TPT}=$ tiempo parcial de tromboplastina (s); F = fibrinógeno $(\mathrm{mg} / \mathrm{dL})$.

encuentra en periodo reproductivo, así como del tiempo que haya transcurrido desde que utilizó su veneno por última vez, de si lo utilizó para alimentarse o para defenderse y del grado de amenaza con que consideró a su potencial agresor.

Por otra parte, el veneno de los vipéridos es una mezcla compleja de diversas enzimas y otros elementos que pueden estar presentes en varias de las especies, presentando ciertas variaciones en cuanto a su composición y concentración. Entre los componentes más comunes encontramos metaloproteinasas, fosfolipasas, aminoácido oxidasa, proteínas anticoagulantes, lectinas y desintegrinas, que al estar presentes en la mayoría de las especies referidas, promueven una reactividad cruzada ante el faboterápico antiviperino, el cual se elabora a partir del veneno de especies seleccionadas de Bothrops sp. y Crotalus sp., pero que resulta efectivo contra el envenenamiento por todas las especies de la familia Viperidae. Por lo anterior, aun cuando no es empleado el veneno de $C$. ravus para la elaboración del antiveneno, por reactividad cruzada resulta efectivo para tratar el envenenamiento provocado por esta especie, tal como ha sido demostrado por laboratorio y en los casos aquí presentados. ${ }^{11}$

Por último, son limitados los casos reportados en donde se ha logrado la identificación del espécimen agresor, y en el caso de C. ravus no se encontraron reportes en la literatura, lo cual quizá esté relacionado con que se trata de una especie endémica de México, con una limitada distribución en la región centro del país, aunque cabe señalar que el reporte de avistamientos sugiere que sí podrían registrarse accidentes que no han sido reportados o que no han sido atribuidos a esta especie.

\section{REFERENCIAS}

1. Walteros D, Paredes A, León NLJ. Accidente ofídico. Protocolo de vigilancia en salud pública. Instituto Nacional de Salud de Colombia. 2014 [Acceso 09 de diciembre de 2020]. Disponible en: http:// manizalessalud.net/wp-content/uploads/2017/11/PRO-AccidenteOfidico.pdf

2. Organización Mundial de la Salud. Mordeduras de serpientes venenosas. 2019 [Acceso 09 de diciembre de 2020]. Disponible en: https://www.who.int/es/news-room/fact-sheets/detail/snakebiteenvenoming

3. Luna-Bauza ME, Martínez-Ponce G, Salazar HAC. Mordeduras por serpiente. Panorama epidemiológico de la zona de Córdoba, Veracruz. Rev Fac Med UNAM. 2004; 47 (4): 149-153.

4. Dirección General de Epidemiología. Anuario de morbilidad general 2019. Secretaría de Salud. Gobierno de México. [Acceso 10 de noviembre de 2020] Disponible en: https://epidemiologia.salud.gob. $\mathrm{mx} /$ anuario/html/morbilidad nacional.html

5. World Health Organization. Guidelines for the production, control and regulation of snake antivenom immunoglobulins. 
WHO; 2018 [Access November 12, 2020]. Available in: https://www.who.int/bloodproducts/snake_antivenoms/ snakeantivenomguide/en/

6. Dirección General de Epidemiología (DGE). Anuario de morbilidad general 2018. Secretaría de Salud. Gobierno de México. 2018 [Acceso 13 de diciembre de 2020]. Disponible en: https:// epidemiologia.salud.gob.mx/anuario/html/anuarios.html

7. Medina TI, Soto Serrano G, Vázquez VG. Comportamiento clínico y epidemiológico de las intoxicaciones por mordedura de serpiente en el Estado de México durante el 2018. Boletín Epidemiológico. Sistema Nacional de Vigilancia Epidemiológica. Dirección General de Epidemiología (DGE). 2019; 48 (36): 3-12.

8. Aguilar MX, Casas AG, Cárdenas RP, Cantellano de RE. Análisis espacial y conservación de los anfibios y reptiles del Estado de México. Ciencia Ergo Sum. 2009; 16 (2): 171-180.
9. Naturalista. Reptiles en Huixquilucan. [Acceso 17 de noviembre de 2020] Disponible en: https://www.naturalista.mx/places/37051\#taxon=26036

10. Instituto Bioclón. Antivipmyn (faboterápico polivalente antiviperino). Información para prescribir. Laboratorios Silanes. México: 2014.

11. Gallegos FG. Caracterización de la reactividad cruzada del faboterápico polivalente antiviperino contra el veneno de serpientes mexicanas del género Crotalus, especies $C$. basiliscus, $C$. atrox, $C$. ravus y C. molossus [Tesis]. Instituto Nacional de Salud Pública; México: 2008 [Acceso 18 de diciembre de 2020]. Disponible en: http://www.catalogoinsp.mx/files/tes/052550.pdf

Conflicto de intereses: Los autores niegan tener cualquier conflicto de intereses. 